\title{
Overexpression of cold-inducible wheat galactinol synthase confers tolerance to chilling stress in transgenic rice
}

\author{
Etsuo Shimosaka*1) and Kenjirou Ozawa ${ }^{2)}$ \\ 1) Crop Breeding Research Division, NARO Hokkaido Agricultural Research Center, 1 Hitsuji-ga-oka, Toyohira-ku, Sapporo, Hokkaido \\ 062-8555, Japan \\ 2) Genetically Modified Organism Research Center, National Institute of Agrobiological Sciences, 2-1-2 Kannondai, Tsukuba, Ibaraki \\ 305-8602, Japan
}

\begin{abstract}
Galactinol synthase (GolS) is considered to be a key regulator of the biosynthesis of Raffinose family oligosaccharides (RFOs). Accumulation of RFOs has been reported to play a role in protection against abiotic stresses. We identified two cDNAs encoding galactinol synthase from wheat (Triticum aestivum L.), which we designated as TaGolS1 and TaGolS2. Expression of the two TaGolS genes was induced by cold stress but not by drought, heat stress or ABA treatment in wheat. We generated transgenic lines of rice (Oryza sativa L.) constitutively overexpressing TaGolS1 or TaGolS2. These transgenic plants accumulated significantly higher levels of galactinol and raffinose than did wild-type plants and exhibited enhanced cold-stress tolerance. The results demonstrate the involvement of galactinol and raffinose in the development of chilling stress in rice and indicate that the genetic modification of the biosynthesis of RFOs by transformation with Gols genes could be an effective method for enhancing chilling-stress tolerance in rice.
\end{abstract}

Key Words: galactinol synthase, wheat, galactinol, raffinose, chilling tolerance, transgenic rice.

\section{Introduction}

Rice (Oryza sativa L.), which was originally cultivated in tropical and subtropical region, suffers chilling injury at temperatures below $15^{\circ} \mathrm{C}$ (Graham and Patterson 1982, Howarth and Ougham 1993). The cultivation area of rice in Japan has been expanded to Hokkaido, the northernmost island of Japan, over the past 300 years. Low temperatures in Hokkaido have often had serious effects on rice production, which have been categorized into two types (Nishiyama 1985): 1) loss of yield due to delay of growth caused by low temperatures during the vegetative stage and 2) coldinduced male sterility caused by low temperatures at the booting stage. To control cold damage, improvements in crop management systems based on an understanding of responses of rice plant to low temperatures have been made and genetic modifications of rice plants to enhance their cold tolerance have also been achieved by breeding.

For genetic improvement in the low temperature tolerance of rice, seed emergence stage and early growth stage have also been targeted in recent breeding programs, be-

Communicated by T. Nishio

Received February 2, 2015. Accepted July 14, 2015.

*Corresponding author (e-mail: etsuo@affrc.go.jp) cause tolerance at these stages is required for stable rice production in a direct seeding system, which has been rapidly spreading in Hokkaido. Previous studies have revealed genetic loci and QTLs responsible for low temperature tolerance. Fujino et al. (2008) identified $q L T G 3-1$ as a QTL controlling low temperature germinability in the seed emergence stage. Regarding seed establishment at the seedling stage under low temperature conditions, genetic variation among rice cultivars has been reported (Bosetti et al. 2012, Ogiwara and Terashima 2001), and QTLs affecting seed establishment at $15^{\circ} \mathrm{C}$ have also been reported (Xie et al. 2014). Besides low temperature tolerance at the germination and seedling stages, it is thought that tolerance to chilling injury at temperatures below $12^{\circ} \mathrm{C}$ at the early seedling stage has to be taken into consideration in a breeding program under the climate conditions in Hokkaido, where air temperature often drops down to a level for inducing chilling damage in late spring.

It has been reported that japonica rice is more tolerant to chilling injury than is indica rice and that genetic variation of the tolerance exists among japonica rice (Baruah et al. 2009). Furthermore, QTLs conferring tolerance have been identified (Lou et al. 2007, Zhang et al. 2005). In addition to reports on mode of the inheritance of chilling stress tolerance, several research groups have shown the possibility of 
conferring chilling tolerance to rice by controlling coldinduced gene expression. Sato et al. (2001) reported that the chilling tolerance of rice was enhanced by overexpression of the rice APX (ascorbate peroxidase) gene. Ozawa et al. (2006) reported that overexpression of wheat UCP (uncoupling protein) led to a significant increase in tolerance to cold treatment at $5^{\circ} \mathrm{C}$ compared to that of wild-type control plants. Liu et al. (2007) showed that the chilling tolerance of rice was enhanced by overexpression of rice the COIN (cold-inducible zinc finger protein) gene. Kawakami et al. (2008) reported that rice transgenic plants in which wheat fructan-synthesizing genes have been introduced exhibited enhanced chilling tolerance. Fructan is one of the storage carbohydrates and is thought to have a function as an osmoprotectant that protects plant cells from drought and cold stresses (Bohnert et al. 1995). Wheat and temperate grasses accumulate fructan during cold acclimation (Yoshida and Tamura 2011), whereas raffinose family oligosaccharides (RFOs) such as raffinose and stachyose are accumulated in legume plants including alfalfa (Medicago sativa L.) as osmoprotectants during cold acclimation, and cold hardy alfalfa cultivars accumulate high levels of raffinose and stachyose compared to the levels in tender cultivars (Castonguay et al. 1995). In rice plants, the amounts of raffinose as well as sucrose, glucose, and fructose increase in response to cold and high-salt stresses (Ito et al. 2006, Morsy et al. 2007, Saito and Yoshida 2011). Furthermore, Morsy et al. (2007) reported that chilling stress increased raffinose content in rice seedlings of a chilling-tolerant genotype, whereas the content decreased in the case of a chilling-sensitive genotype, suggesting that RFOs (Raffinose family oligosaccharides) have a role in the development of chilling tolerance in rice. The biosynthesis of RFOs proceeds by the transfer of galactose units from galactinol to sucrose catalyzed by raffinose synthase (RS, EC 2.4.1.82), generating trisaccharide raffinose. Raffinose is subsequently used as a substrate for the synthesis of tetrasaccharide stachyose by the enzyme stachyose synthase (StS, EC 2.4.1.67). Galactinol is the galactosyl donor for biosynthesis of RFOs and is formed from UDP-galactose and myo-inositol catalyzed by galactinol synthase (GolS, EC 2.4.1.123), which is considered a key regulator in the RFO metabolic pathway (Keller and Pharr 1996, Peterbauer and Richter 2001).

Considering the effects of overexpression of wheat fructansynthesizing genes, sucrose:sucrose 1-fructosyltransferase (1SST, EC 2.4.1.99) and sucrose:fructan 6-fructosyltransferase (6-SFT, EC 2.4.1.10), on the enhancement of rice chilling tolerance by the accumulation of fructose-based oligo- and polysaccharides (Kawakami et al. 2008), it would be interesting to know if rice plants overexpressing alien GolS genes accumulate higher levels of RFOs which enhance chilling tolerance in rice seedlings. This is the first report on molecular cloning of wheat GolS genes and enhancement of rice chilling tolerance by overexpression of wheat GolS genes.

\section{Materials and Methods}

\section{Plant material and EST clones}

Winter wheat (Triticum aestivum L.) cultivar 'Valuevskaya', which exhibits a high level of freezing tolerance (Yoshida et al. 1998), was used for expression analysis of GolS genes. The rice cultivar 'Yukihikari', which is chilling sensitive in the seedling stage and grown in Hokkaido, was used for the production of transgenic rice (Rice cultivar database: http://ineweb.narcc.affrc.go.jp). Developed transgenic lines were evaluated for their contents of RFOs and responses to stress treatments. Wheat EST clones (Mochida et al. 2006) used for cloning GolS genes were provided by Kihara Institute for Biological Research, Yokohama City University (Yokohama, Japan).

\section{Molecular cloning of galactinol synthase genes in wheat}

The wheat galactinol synthase (GolS) coding sequences were predicted from the wheat EST database which derived from various stress-treated cDNA libraries (Mochida et al. 2006), based on the deduced amino acid sequences of Arabidopsis thaliana GolS cDNAs (accession No. AB062848 for AtGolS1; Taji et al. 2002) by using the BLAST program (Karlin and Altschul 1993). The BLAST search identified two stress-inducible GolS genes in EST clones obtained from cold-acclimated plants (cv. Valuevskaya); the two genes were referred to as TaGolS1 and TaGolS2. Nucleotide sequences of the GolS genes were determined by using the Li-COR 4200LD-G sequencer (Li-COR, Lincoln, NE, USA). Amino acid sequences were aligned using the GENETYXMAC program (Software Development,Tokyo, Japan). A data set consisting of the deduced amino acid sequences from TaGolS1 and TaGolS2 cDNAs and 16 GolSs members from a wide variety of plant species was subjected to phylogenetic analysis: rice, maize, Arabidopsis thaliana L., soybean, zucchini, common bugle (Ajuga reptans L.), alfalfa (Medicago sativa L.), Medicago falcate L., muskmelon (Cucumis melo L.) and common buckwheat. A phylogenetic tree was constructed by the Neighbor-Joining clustering method (Saitou and Nei 1987).

The nucleotide sequences reported have been submitted to EMBL, GenBank and DDBJ under the accession numbers AB250356 (TaGolS1) and AB250357 (TaGolS2).

\section{RNA isolation and expression analysis}

Total RNA was isolated from plant samples using TRIzol reagent (Invitrogen, Carlsbad, CA, USA) according to the manufacturer's instructions. Ten $\mu \mathrm{g}$ of total RNA was separated in $1.4 \%(\mathrm{w} / \mathrm{v})$ agarose gel containing $0.5 \%(\mathrm{v} / \mathrm{v})$ formaldehyde and transferred onto a nylon filter using standard procedures (Sambrook et al. 1989). Hybridization was performed at $65^{\circ} \mathrm{C}$ for $1 \mathrm{~h}$ in Rapid-Hyb Buffer (GE Healthcare Bio-Sciences, Piscataway, NJ, USA) with addition of a ${ }^{32} \mathrm{P}$-labeled probe. The probes were PCR-amplified from pBluescripts II SK- plasmids (Stratagene, La Jolla, CA, USA) containing TaGolS cDNAs with primers for cDNA- 
specific fragments: TaGolS1-specific primers (forward 5'-TGCATAGGCTCGCTCGCTG-3' and reverse T7 primer on a vector) and TaGolS2-specific primers (forward 5'GCCATCAGCTGCGTAGTC-3' and reverse T7 primer on a vector) or primers for a common fragment between TaGolSI and TaGols2: (forward 5'-ATGGCTCCCATGCTCAAG-3' and reverse 5'-TGTGGAGCTGGATGTTCTC-3'). The cDNA-specific fragments including 250 bases and 281 bases of the 3'UTR of TaGolS1 and TaGolS2, respectively, were used for detection of gene-specific transcripts. The cDNA-common fragment including 739 bases of the coding sequence of TaGolSl was also used for Northern analysis. Following hybridization, filters were washed once for 20 min with $2 \times \mathrm{SSC}$ and $0.1 \%$ SDS at room temperature and twice for $15 \mathrm{~min}$ with $1 \times \mathrm{SSC}$ and $0.1 \% \mathrm{SDS}$ at $65^{\circ} \mathrm{C}$. Total RNA $(1 \mu \mathrm{g})$ was reverse transcribed by mRNA Selective PCR Kit (TaKaRa, Tokyo, Japan). RT-PCR (reverse transcription-polymerase chain reaction) was performed with specific primers: TaGolSl-specific primers (forward 5'-TGCATAGGCTCGCTCGCTG-3' and reverse 5'-GAT TGATCTACAATACCAAAACGTGTAG-3') and TaGols2specific primers (forward 5'-GCCATCAGCTGCGTAGTC$3^{\prime}$ and reverse 5'-CCATGCATACGTACGTATAGCAG-3') or primers: OsUbiquitin (forward 5'-CCAGGACAAGAT GATCTGCC-3' and reverse 5'-AAGAAGCTGAAGCATCC AGC-3') as a control (Thao et al. 2007).

\section{Stress treatments of wheat plants}

Wheat plants were subjected to various kinds of stress treatment to observe the expression of GolS genes. The wheat plants were grown at $20 / 15^{\circ} \mathrm{C}$ (day/night) in a controlled environment for 7 days as previously described (Shimosaka et al. 1999). Control plants at the two-leaf stage were maintained under the same conditions. For cold treatment, plants at the two-leaf stage were exposed to a temperature of 0,4 or $8^{\circ} \mathrm{C}$ for $24 \mathrm{~h}$ with an $8 \mathrm{~h}$ photoperiod $\left(50 \mu \mathrm{mol} \mathrm{m} \mathrm{m}^{-2} \mathrm{~s}^{-1}\right)$. Drought stress was induced by withholding water supply for 5 days, by which time the plants were visibly wilted. Heat shock treatment was performed by incubating plants at $42^{\circ} \mathrm{C}$ for $3 \mathrm{~h}$. Plants subjected to treatment with $\mathrm{ABA}$ and high-salt stress were grown hydroponically in solutions containing $50 \mu \mathrm{M}$ ABA (mixed isomers, Sigma-Aldrich, St. Louis, MO, USA) and $400 \mathrm{mM} \mathrm{NaCl}$, respectively, for $24 \mathrm{~h}$.

\section{Transformation of rice plants}

For the production of transgenic rice plants (Oryza sativa L. cv. Yukihikari), XbaI-KpnI fragments containing TaGolSI or TaGolS2 were fused in sense orientation to the $X b a \mathrm{I}-$ KpnI site of pMSH1, a plant binary vector (Kawasaki et al. 1999). The TaGols fragment was placed under the control of the cauliflower mosaic virus (CaMV) 35S constitutive promoter (Fig. 3A). The recombinant vectors pMSHTaGolS1 and TaGolS2 were introduced into Agrobacterium tumefaciens strain EHA101 (Hood et al. 1986), and then transgenic rice plants were obtained by means of
Agrobacterium-mediated transformation according to the previously reported protocol (Toki 1997).

\section{Cold treatment for evaluation of chilling-stress tolerance}

Rice seeds were imbibed for 3 days at $25^{\circ} \mathrm{C}$ and sown in a water-saturated commercial potting medium (Katakura Chikkarin, Tokyo, Japan) in plastic dishes. They were grown in a greenhouse at $23-29^{\circ} \mathrm{C}$ for 14 days until the three-leaf stage and then exposed to a temperature of $5^{\circ} \mathrm{C}$ under continuous light at $12 \mu \mathrm{mol} \mathrm{m}^{-2} \mathrm{~s}^{-1}$ for 14 days, while control plants at the two-leaf stage were maintained under the same conditions. After the cold treatment, the plants were regrown in a greenhouse for 14 days. The seedlings were evaluated for their percentage survival based on observations (Ozawa et al. 2006, Sato et al. 2001). Each treatment consisting of 12 seedlings was repeated four times.

\section{Carbohydrate analysis}

Galactinol, raffinose and stachyose contents in matured leaves and third leaves after cold treatment of transgenic rice plants were determined by high-performance anion exchange chromatography (HPAEC) analysis. Finely chopped leaves excised from rice plants were boiled in boiling deionized water for $1 \mathrm{~h}$ to extract total water-soluble sugars. The extract solution was filtered through $0.45 \mu \mathrm{m}$-pore-sized membrane filter. HPAEC analysis was performed by using a DX500 gradient chromatography system coupled with pulsed amperometry detection by using an ED40 detector (Dionex, Sunnyvale, CA, USA) equipped with a CarboPac PA-1 (4 $\times 250 \mathrm{~mm})$ analytical column and a CarboPac PA-1 guard column $(4 \times 50 \mathrm{~mm})$. For the separation of galactinol, the sample solution was eluted at $1 \mathrm{ml} \mathrm{min}^{-1}$ in $100 \mathrm{mM}$ $\mathrm{NaOH}$ for $2 \mathrm{~min}$ and then with a linear gradient of $0-100 \mathrm{mM}$ sodium acetate in $100 \mathrm{mM} \mathrm{NaOH}$ for $18 \mathrm{~min}$. For the separation of raffinose and stachyose, the sample solution was eluted at $1 \mathrm{ml} \mathrm{min}{ }^{-1}$ in $150 \mathrm{mM} \mathrm{NaOH}$ for $2 \mathrm{~min}$ and then with a linear gradient of 0-40 mM sodium acetate in $150 \mathrm{mM} \mathrm{NaOH}$ for $18 \mathrm{~min}$. Each sugar was identified and quantified by the external standard method using authentic galactinol, raffinose and stachyose.

\section{Results}

Identification and sequence analysis of wheat cDNAs encoding GolS

In the wheat EST database, two cDNA clones in the library derived from cold-treated wheat seedlings were found to show significant similarity to an Arabidopsis GolS cDNA (accession No. AB062848 for AtGolS1). The expectation value threshold is $1.0 \mathrm{E}+1$ for the similarity searches. They were designated as TaGolS1 and TaGolS2. The TaGolS1 and TaGolS2 cDNAs contained 960-bp and 972-bp open reading frames that encode polypeptides of 319 and 323 amino acids, respectively. The two TaGolS cDNAs share $90 \%$ identity at the nucleotide level and are $96 \%$ similar at the protein level, and they differ from each other by only 12 amino acid 
residues. Deduced TaGolS proteins had most similarity to rice WSI76 protein (accession No. BAA05538), which is believed to be the rice GolS (Liu et al. 1998, Takahashi et al. 1994), sharing $81 \%$ and $82 \%$ identify with TaGolS1 and TaGolS2 proteins, respectively, in the GenBank/EMBL, PIR, and SwissProt databases.

Amino acid sequences of TaGolS1 and TaGolS2 were compared with those of maize (Zea mays L.) (ZmGolS1, accession No. AAQ07248; Zhao et al. 2004), Arabidopsis thaliana L. (AtGolS1, accession No. BAB78530; Taji et al.
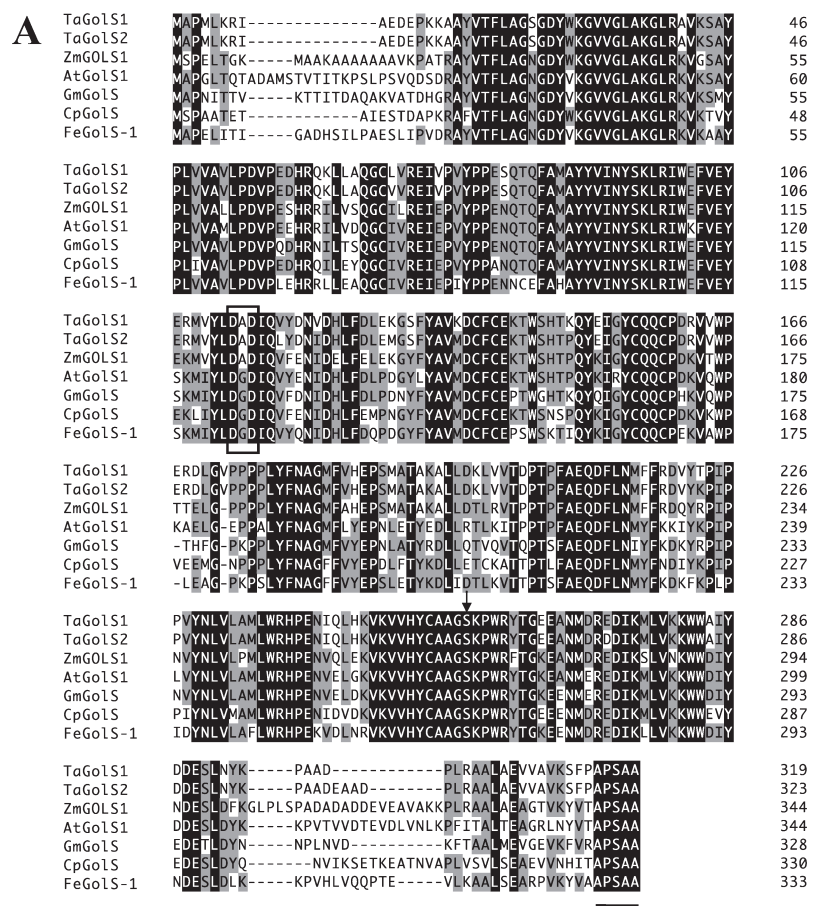

B

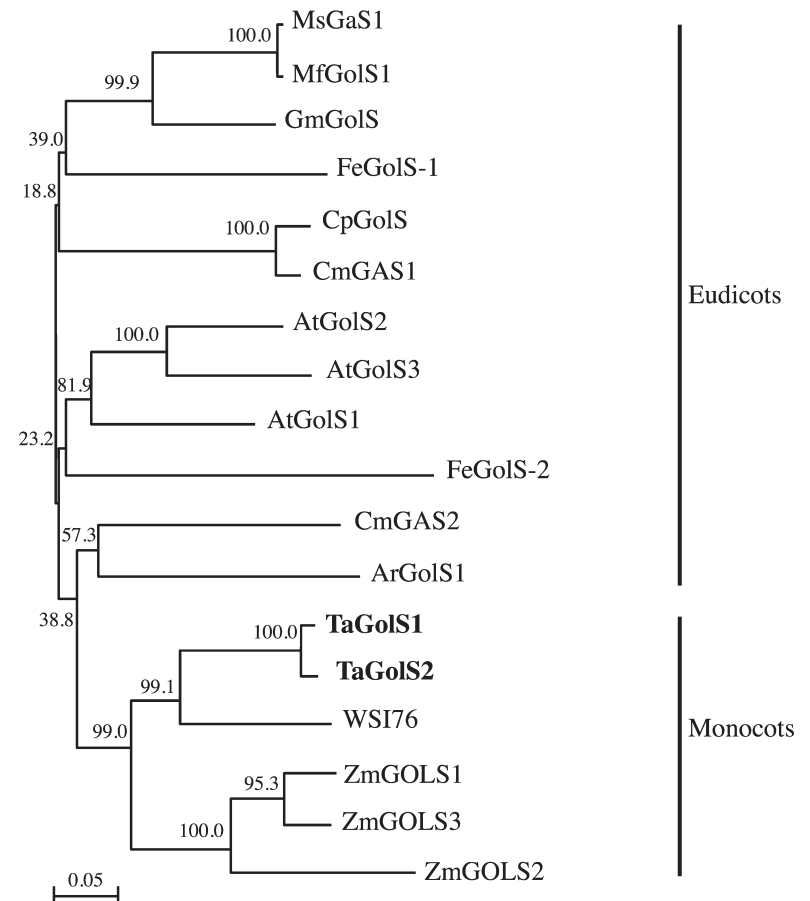

2002), soybean (Glycine max L.) (GmGolS1accession No. AAM96867; Obendorf et al. 2004), zucchini (Cucurbita pepo L.) (U.S. Patent 5648210 for CpGolS; Kerr et al. 1997) and common buckwheat (Fagopyrum esculentum Moench.) (FeGolS-1, accession No.AAM96870; Ueda et al. 2005) for which their galactinol synthetic capacities when expressed as recombinant proteins in E.coli have been reported. Both TaGolS1 and TaGolS2 proteins share a high degree of amino acid sequence similarity with other GolSs (Fig. 1A). ZmGOLS1 shows high homology of $82 \%$ and $78 \%$ with TaGolS1 and TaGolS2 proteins, respectively. Those of eudicot plants (AtGolS1, GmGolS, CpGolS, FeGols-1) show $69 \%-79 \%$ identity to TaGolS1 and TaGolS2 proteins. Alignment analysis of the GolSs revealed the presence of a conserved domain, YLDA/GDIQ, including a small common motif, DXD, in all GolSs examined. The $\mathrm{D} X \mathrm{D}$ motif is thought to be involved in the binding of manganese ions and to be conserved in most galactosyltransferases and essential for their enzyme activity (Breton et al. 1998, Busch et al. 1998). The putative serine phosphorylation site and the carboxyl terminal pentapeptide, APSAA (Sprenger and Keller 2000), are also exhibited in all GolSs examined.

An unrooted phylogenetic tree constructed from a data set of 16 GolSs members including the five GolSs described above is shown in Fig. 1B. Relationships shown in this cladogram generally reflect standard groupings of monocots

Fig. 1. Alignment and phylogenetic tree analysis of GolS amino acid sequences from various plant species. A. Comparison of the deduced amino acid sequence of TaGolS cDNAs with other members of GolSs: maize (accession No. AAQ07248 for ZmGOLS1; Zhao et al. 2004), Arabidopsis thaliana (accession No. AB062848 for AtGolS1; Taji et al. 2002), soybean (accession No. AAM96867 for GmGolS; Obendorf et al. 2004), zucchini (U.S. Patent 5648210 for CpGolS; Kerr et al. 1997), common buckwheat (accession No.AAM96870 for FeGolS-1; Ueda et al. 2005). Amino acids conserved in at least four members are shown on a gray background, and highly conserved amino acids are highlighted. The common motif believed to be essential for the enzyme activity of galactosyltransferases, $\mathrm{D} X \mathrm{D}$, is boxed. The arrow shows the putative serine phosphorylation site. The carboxyl terminal pentapeptide (APSAA) is underlined. B. A phylogenetic tree derived from the deduced amino acid sequence of GolSs: rice (accession No. BAA05538 for WSI76; Takahashi et al. 1994), maize (ZmGOLS1, accession No. AAQ07249 and AAQ07250 for ZmGOLS2 and 3; Zhao et al. 2004), Arabidopsis thaliana L. (AtGolS1, accession No. BAB78530, BAB785301 and BAB78532 for AtGolS2 and 3; Taji et al. 2002), soybean (GmGolS), zucchini (CpGolS), common bugle (accession No. CAB51533 for ArGolS1; Sprenger and Keller 2000), alfalfa (accession No. AAM97493 for MsGaS1; Cunningham et al. 2003), muskmelon (accession No. AAL78687 for CmGAS1; Volk et al. 2003), common buckwheat (FeGolS-1, accession No.AAM96868 for FeGolS-2; Ueda et al. 2005), Medicago falcate L. (accession No. ACM50915 for MfGolS1; Zhuo et al. 2013) and wheat (accession No. BAF51565 and BAF51566 for TaGolS1 and 2; in this report). The tree was drawn by the Neighbor-Joining method. Numbers on each branch represent bootstrap percentages (based on 1000 replicates). The scale bar indicates distance value of 0.05 substitutions per site. 


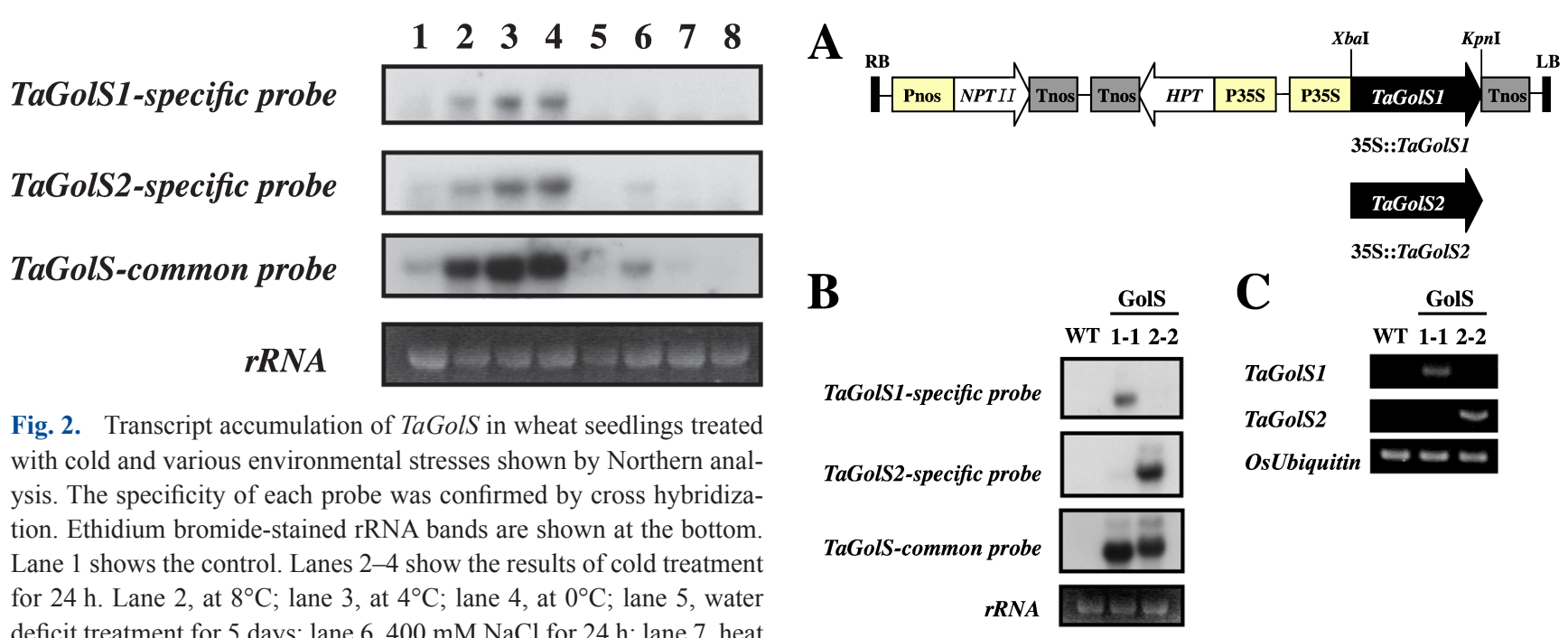

deficit treatment for 5 days; lane $6,400 \mathrm{mM} \mathrm{NaCl}$ for $24 \mathrm{~h}$; lane 7 , heat shock at $42^{\circ} \mathrm{C}$ for $3 \mathrm{~h}$; lane $8,50 \mu \mathrm{M}$ ABA for $24 \mathrm{~h}$.

and eudicots species. TaGolS1 and TaGolS2 were closely related to the rice predicted GolS 'WSI76' protein and in a clade of monocots (Fig. 1B).

\section{Expression of TaGolS1 and TaGolS2 in wheat plants} under cold and various environmental stresses

Total RNAs were purified from wheat plants at the twoleaf stage that had been treated with various stresses. Northern hybridization was conducted using TaGolS1 or TaGolS2 cDNA-specific fragments and a TaGolS common fragment as hybridization probes (Fig. 2). The specificity of each specific probe was verified by performing cross-hybridization (data not shown). A TaGolS-common fragment amplified from TaGolS1 cDNA shows high homology of 99\%, 92\% and $86 \%$ with TaGols2, tplb0053i13 and RFL_Contig1692, respectively. The tplb0053i13 and RFL_Contig1692 are full-length cDNAs of wheat might be encoding the other wheat-GolS. In the control plants grown at $20^{\circ} \mathrm{C}$, each TaGolS transcript was detected at a low basal level (Fig. 2, lane 1). In contrast, the transcript levels of TaGolS1, TaGolS2 and TaGolSs were crearly high after twenty-four hours of exposure to a low temperature compared to the control, which were observed in the order of $8^{\circ} \mathrm{C}<4^{\circ} \mathrm{C}$ $<0^{\circ} \mathrm{C}$ (Fig. 2, lanes 2-4). Salt stress with $400 \mathrm{mM} \mathrm{NaCl}$ also slightly increased TaGolS mRNAs (Fig. 2, lane 6). Water deficit treatment to drought stress, heat shock at $42^{\circ} \mathrm{C}$ and $50 \mu \mathrm{M}$ ABA application had no effect on TaGolSs transcript levels (Fig. 2, lanes 5, 7 and 8).

\section{Expression of TaGolS genes and accumulation of galactinol} and raffinose in transgenic rice plants

To examine whether TaGolS encodes the GolS protein with a galactinol synthetic capacity in rice, we generated transgenic rice plants overexpressing TaGolS1 or TaGolS2 under control of the constitutive CaMV $35 \mathrm{~S}$ promoter (35S::TaGolS1 and 35S::TaGolS2; Fig. 3A). The line

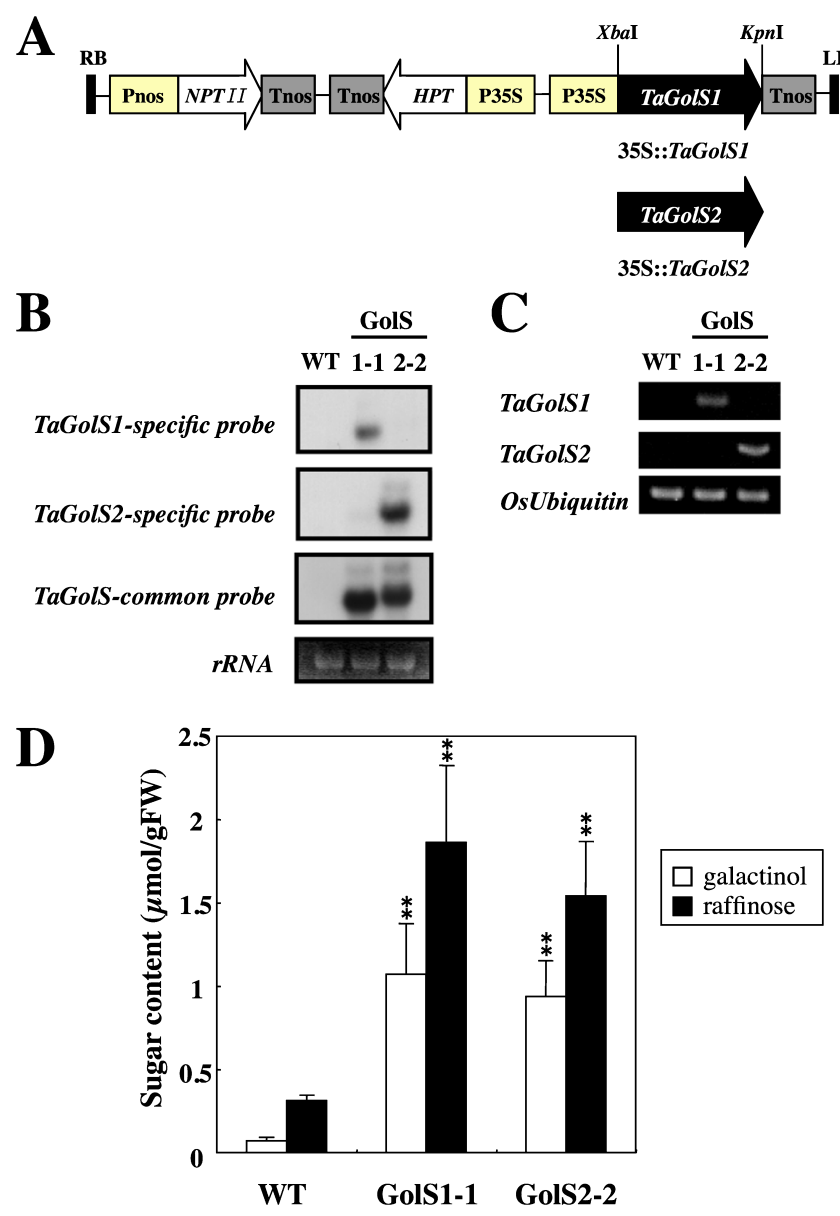

Fig. 3. Analysis of transgenic rice lines overexpressing TaGolS. A. Schematic representation of the construct used to overexpress TaGolS1 or TaGolS2 in rice. Pnos, promoter sequence of nopaline synthase gene; NPTII, neomycine phosphotransferase gene; Tnos, terminator sequence of nopaline synthase gene; HPT, hygromycin B phosphotransferase gene; P35S, promoter sequence of the cauliflower mosaic virus (CaMV)35S; TaGolS1 and TaGolS2, galactinol synthase cDNA from wheat; RB, right T-DNA border; LB, left T-DNA border. B. Expression of TaGolS1 and TaGolS2 in transgenic rice lines ( $\mathrm{T}_{1}$ generation) determined by Northern analysis. Ethidium bromide-stained rRNA bands are shown at the bottom. C. Specific expression of TaGolS1 and TaGolS2 in transgenic rice lines ( $\mathrm{T}_{1}$ generation) determined by RT-PCR analysis. D. Galactinol and raffinose contents of transgenic rice lines overexpressing TaGolS1 or TaGolS2. Mature leaves were sampled from wild-type (WT) and two independent transgenic lines (GolS 1-1 and GolS 2-2: $\mathrm{T}_{1}$ generation). Each data point represents the mean \pm standard deviation from four individual plants. Bars labeled with asterisks show significant differences between WT and transgenic plants by Welch's $t$-test at ** $\mathrm{P}=0.01$.

GolS1-1 ( $\mathrm{T}_{1}$ generation) with introduced TaGolS1 was selected as the line showing the highest content of raffinose in mature leaves from eighteen independently transformed lines. For TaGolS2, the line GolS 2-2 was selected in the same manner. Northern analysis confirmed expression of the transgene in the two transgenic lines in mature leaves, whereas no expression was observed in the wild-type 
galactinol

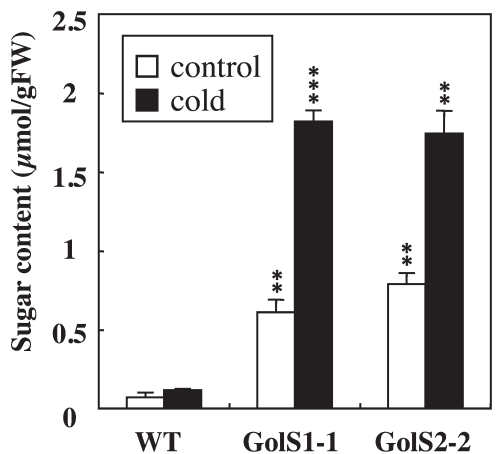

raffinose

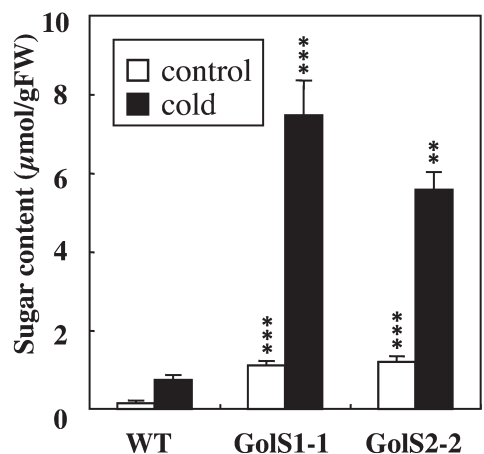

stachyose

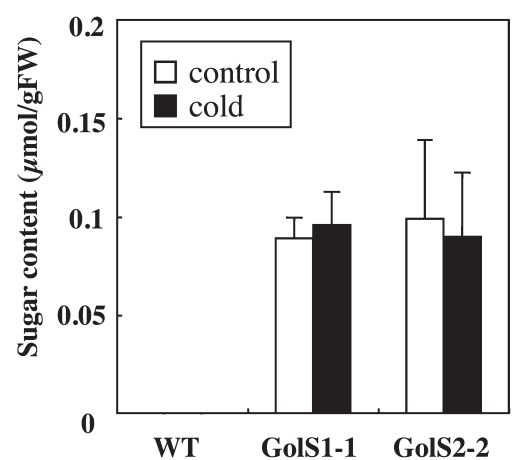

Fig. 4. Galactinol, raffinose and stachyose contents of transgenic rice lines overexpressing TaGolS1 or TaGolS2 after cold treatment. Fourteenday-old plants of wild-type (WT) and two independent transgenic lines (GolS 1-1 and GolS 2-2: $\mathrm{T}_{2}$ generation) were subjected to cold at $5^{\circ} \mathrm{C}$ under continuous light for 14 days. The third leaves were sampled from WT, GolS 1-1 and GolS 2-2 transgenic plants. Each data point represents the mean \pm standard deviation from four individual plants. Bars labeled with asterisks show significant differences between WT and transgenic plants by Welch's $t$-test at $* * \mathrm{P}=0.01$ and $* * * \mathrm{P}=0.001$.

control plant in spite of the fact that the TaGolS-common fragment as a probe shows high homology of $91 \%$ and $92 \%$ with two rice GolS genes, Os07g0687900 (wsi76) and Os03g0316200 (OsGolS1), respectively (Fig. 3B). Additionally, RT-PCR analysis also confirmed specific expression of the transgene in transgenic plants by the specific primer sets of TaGolS1 and TaGolS2 (Fig. 3C). Galactinol and raffinose contents in mature leaves of wild-type and transgenic rice plants are shown in Fig. 3D. Stachyose was not detected in either transgenic or wild-type plants. GolS11 and GolS2-2 plants accumulated galactinol and raffinose at high levels compared to the levels in wild-type plants. Galactinol contents in the GolS1-1 and GolS2-2 transgenic plants were 1.1 and $0.9 \mu \mathrm{mol} \mathrm{g}^{-1}$ fresh weight, respectively which were 15.1- and 13.2-fold higher than that in wildtype plants. Raffinose contents in those transgenic plants were 1.9 and $1.5 \mu \mathrm{mol} \mathrm{g}^{-1}$ fresh weight, which were 6.0 and 4.9-fold higher than that in wild-type plants. The $\mathrm{T}_{2}$ progenies of these transgenic plants (GolS1-1 and GolS2-2) with confirmed the expression of TaGolS1 and TaGols2 were used for subsequent experiments including the evaluation of chilling stress tolerance.

Accumulation of galactinol, raffinose and stachyose and chilling-stress tolerance in transgenic rice seedlings after cold treatment

Transgenic rice plants were examined for their galactinol, raffinose and stachyose contents in the leaves of 14days old seedlings subjected to cold treatment, followed by evaluation of chilling stress tolerance. Fig. 4 shows galactinol, raffinose and stachyose contents in transgenic and wild-type plants. The mean galactinol content in wild-type plants was $0.1 \mu \mathrm{mol} \mathrm{g}^{-1}$ fresh weight after cold treatment, which was 1.7-fold higher than that in control conditions. The mean galactinol contents in GolS1-1 and GolS2-1 transgenic lines after cold treatment were 1.8 and $1.7 \mu \mathrm{mol} \mathrm{g}{ }^{-1}$ fresh weight, respectively, which were 3.3- and 2.2-times higher than that in control conditions. The mean raffinose content in wild-type plants was $0.7 \mu \mathrm{mol} \mathrm{g}^{-1}$ fresh weight after cold treatment, which was 4.9 -fold higher than that in control conditions. The mean raffinose contents in GolS1-1 and GolS2-1 transgenic lines after cold treatment were 7.5 and $5.6 \mu \mathrm{mol} \mathrm{g}^{-1}$ fresh weight, respectively, which were 6.7and 4.7-times higher than that in control conditions. Stachyose was not detected in wild-type plants. In contrast, stachyose was detected in transgenic rice plants, though its levels were very low, below $0.1 \mu \mathrm{mol} \mathrm{g}^{-1}$ fresh weight. The amount of stachyose in transgenic rice did not show significant difference between control and cold-treated plants.

Fig. 5 shows the chilling tolerance of rice transgenic lines. Most of the wild-type plants showed rolling leaves, a symptom of chilling injury, at day 10 of cold treatment. After completing chilling treatment and subsequent incubation in the greenhouse, the growth of chilling-stressed wild-type plants was severely inhibited, resulting in death (Fig. 5A). The survival rate of wild-type plants was $33.3 \%$ in all replicates (Fig. 5B). In contrast, survival rate of GolS1-1 and GolS2-2 plants were $85.4 \%$ and $80.3 \%$, respectively, indicating their significantly higher levels of chilling tolerance compared to that in wild-type plants.

\section{Discussion}

We analyzed two cDNAs, TaGolS1 and TaGolS2, derived from cold-treated wheat plants. Deduced amino acid sequences from TaGolS1 and TaGolS2 revealed a high similarity to GolS proteins from other plant species (Fig. 1A). Alignment analysis revealed a highly conserved domain, YLDA/GDIQ, with a DXD motif of galactosyltransferases and a carboxyl terminal pentapeptide, APSAA, known as structural features of GolS that exist in both TaGolS1 and TaGolS2 proteins (Breton et al. 1998, Busch et al. 1998, Sprenger and Keller 2000). GolS genes are known to show responses to various stresses including cold, drought, salt 

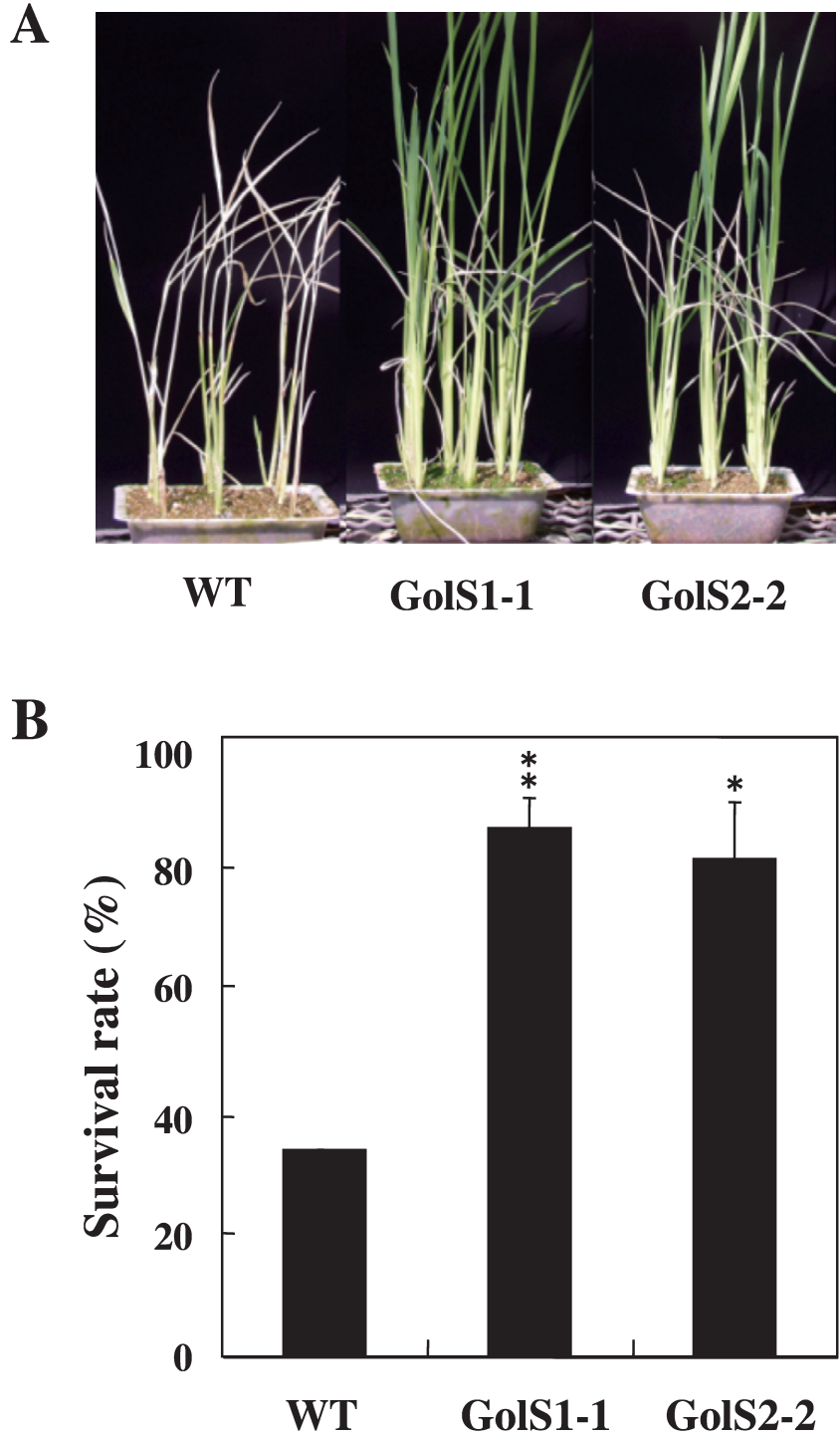

Fig. 5. Comparison of chilling tolerance in wild-type and transgenic rice lines overexpressing TaGolS1 or TaGolS2. Fourteen-day-old plants of wild-type (WT) and two independent transgenic lines (GolS 1-1 and GolS 2-2: $\mathrm{T}_{2}$ generation) were subjected to cold at $5^{\circ} \mathrm{C}$ under continuous light for 14 days. After cold treatment, they were regrown for 14 days at $23-29^{\circ} \mathrm{C}$. A. Chilling tolerance of WT, GolS $1-1$ and GolS 2-2 transgenic plants. B. Survival rate of WT, GolS 1-1 and GolS 2-2 transgenic plants after chilling treatments. Each data point represents the mean \pm standard deviation from three independent experiments. Bars labeled with asterisks show significant differences between WT and transgenic plants by Welch's $t$-test at $* \mathrm{P}=0.02$ and $* * \mathrm{P}=0.01$

and heat (Cunningham et al. 2003, Downie et al. 2003, Panikulangara et al. 2004, Taji et al. 2002, Takahashi et al. 1994, Wang et al. 2009, Zhuo et al. 2013). Both transcript levels of TaGolS1 and TaGolS2 clearly increased in response to cold stress at temperatures below $8^{\circ} \mathrm{C}$ (Fig. 2). The drastic increase in response to cold treatment was more pronounced when using the TaGolS common probe. This observation suggests the presence of other GolS homologue genes in wheat. Indeed, two cDNAs, tplb0053i13 and RFL Contig1692, showed high similarity to TaGols1 and TaGolS2. In contrast, both transcripts of TaGolS1 and $\mathrm{TaGolS} 2$ were not detected under drought stress induced by water deficit treatment, heat shock stress or ABA application. These results were similar to the expression patterns of AtGolS3 in Arabidopsis and MfGolS1 in Medicago falcate L. under an abiotic stress condition (Nishizawa et al. 2008, Taji et al. 2002, Zhuo et al. 2013). In contrast, expression of Os07g0687900 (wsi76) and Os03g0316200 (OsGolS1) was induced by ABA application (Rice Expression Profile database: http:// http://ricexpro.dna.affrc.go.jp), and their riceGolS homologue was expressed especially in anthers of rice. Sequence comparison among GolS proteins and transcriptional data presented in this paper reveal that the TaGolS1 and TaGolS2 genes encode cold-inducible GolS in wheat. Additionally, the transcriptional data suggest that TaGolS1 and TaGolS2 encode the GolS as a key regulator in biosynthesis of RFOs during cold acclimation and that the signal transduction pathway involved in the cold induction of RFOs is not ABA-mediated in wheat (Fig. 2).

In transgenic rice plants overexpressing TaGolS1 or $\mathrm{TaGolS2}$, galactinol, raffinose and stachyose contents were significantly higher than those in wild-type plants under normal and cold conditions (Fig. 3D and Fig. 4), indicating that TaGolS1 and TaGolS2 encode GolS, which acts as a regulator of raffinose synthesis in different plant species. Cold treatment increased the amounts of galactinol and raffinose in both wild-type and transgenic plants, and it is noteworthy that the effects of cold treatment were greater in transgenic plants (Fig. 4). It has been reported that the amount of raffinose increased in response to cold stress in rice (Ito et al. 2006, Morsy et al. 2007, Saito and Yoshida 2011), and it has been suggested that two GolSs and four RSs are responsible for galactinol and raffinose accumulation induced by cold treatment in rice (Saito and Yoshida 2011). This finding also demonstrated that cold-induced expression of $O s R S s$ gene contributed to large accumulation of raffinose by utilization of an excess amount of galactinol as the substrate in transgenic plants after cold treatment. Thus, it is assumed that cold treatment increases the content of UDPgalactose and/or myo-inositol, which are the substrates for galactinol; however, native GolSs and RSs in rice do not metabolize all of them in wild-type plants, whereas transgenic plants overexpressing TaGolS could utilize an excess amount of substrate, resulting in differences in the rate of increase in the accumulation of galactinol and raffinose between wild-type and transgenic plants. Indeed, Morsy et al. (2007) reported that chilling stress increased inositol content in rice seedlings of a chilling-sensitive genotype, while the contents were equal to the control in the case of a chilling-tolerant genotype, suggesting that inositol is metabolized as the substrate for galactinol in a chilling-tolerant genotype. Stachyose was only detected in transgenic seedlings and was not increased by cold treatment. The metabolism regarding StS might not be affected by low temperature. 
Our results revealed that overexpression of TaGolS1 or TaGolS2 in rice plants leads to a significant increase in tolerance to chilling stress (Fig. 5). The enhancement of chilling tolerance could be ascribed to the effect of large accumulation of galactinol and/or RFOs induced by the overexpression of TaGolS1 and TaGolS2. Therefore, genetic engineering of GolS genes could be an alternative way to improve chilling tolerance in rice seedlings.

\section{Acknowledgments}

We wish to thank Prof. Yasunari Ogihara and Dr. Kanako Kawaura (Kihara Institute for Biological Research, Yokohama City University, Yokohama, Japan) for providing the wheat EST clones. We are grateful to Dr. Norio Iriki (NARO Hokkaido Agricultural Research Center, Sapporo, Japan) for helpful discussion and continuous encouragement. We also thank Dr. Midori Yoshida (NARO Hokkaido Agricultural Research Center, Sapporo, Japan) for technical support and helpful discussion in carbohydrate analysis. This work was supported in part by a grant from the Ministry of Agriculture, Forestry and Fisheries of Japan (Research Project for Development of innovative crops through the molecular analysis of useful genes).

\section{Literature Cited}

Baruah,A.R., N. Ishigo-Oka， M.Adachi， Y.Oguma， Y.Tokizono, K. Onishi and Y.Sano (2009) Cold tolerance at the early growth stage in wild and cultivated rice. Euphytica 165: 459-470.

Bohnert, H.J., D.E. Nelson and R.G. Jensen (1995) Adaptations to environmental stresses. Plant Cell 7: 1099-1111.

Bosetti,F., C.Montebelli, A.D.L.C.Novembre, H.P.Chamma and J.B.Pinheiro (2012) Genetic variation of germination cold tolerance in Japanese rice germplasm. Breed. Sci. 62: 209-215.

Breton, C., E.Bettler, D.H.Joziasse, R.A. Geremia and A.Imberty (1998) Sequence-function relationships of prokaryotic and eukaryotic galactosyltransferases. J. Biochem. 123: 1000-1009.

Busch, C., F. Hofmann, J. Selzer, S. Munro, D. Jeckel and K.Aktories (1998) A common motif of eukaryotic glycosyltransferases is essential for the enzyme activity of large clostridial cytotoxins. J. Chem. Biol. 273: 19566-19572.

Castonguay, Y., P. Nadeau, P. Lechasseur and L. Chouinard (1995) Differential accumulation of carbohydrates in alfalfa cultivars of contrasting winterhardiness. Crop Sci. 35: 509-516.

Cunningham, S.M., P.Nadeau, Y.Castonguay, S.Laberge and J.J. Volenec (2003) Raffinose and stachyose accumulation, galactinol synthase expression, and winter injury of contrasting alfalfa germplasms. Crop Sci. 43: 562-570.

Downie, B., S. Gurusinghe, P. Dahal, R.R. Thacker, J.C. Snyder, H. Nonogaki, K.Yim, K. Fukanaga, V.Alvarado and K.J.Bradford (2003) Expression of a GALACTINOL SYNTHASE gene in tomato seeds is up-regulated before maturation desiccation and again after imbibition whenever radicle protrusion is prevented. Plant Physiol. 131: 1347-1359.

Fujino, K., H. Sekiguchi, Y. Matsuda, K. Sugimoto, K. Ono and M. Yano (2008) Molecular identification of a major quantitative trait locus, qLTG3-1, controlling low-temperature germinability in rice. Proc.
Natl. Acad. Sci. USA 105: 12623-12628.

Graham, D. and B.D. Patterson (1982) Responses of plants to low, nonfreezing temperature: proteins, metabolism, and acclimation. Annu. Rev. Plant Physiol. 33: 347-372.

Hood, E.E., G.L.Helmer, R.T.Fraley and M.D. Chilton (1986) The hypervirulence of Agrobacterium tumefaciens A281 is encoded in a region of pTiBo542 outside of T-DNA. J. Bacteriol. 168: 12911301.

Howarth, C.J. and H.J. Ougham (1993) Gene expression under temperature stress. New Phytol. 125: 1-26.

Ito, Y., K. Katsura, K. Maruyama, T.Taji, M.Kobayashi, M. Seki, K. Shinozaki and K. Yamaguchi-Shinozaki (2006) Functional analysis of rice DREB1/CBF-type transcription factors involved in cold-responsive gene expression in transgenic rice. Plant Cell Physiol. 47: 141-153.

Karlin, S. and S.F.Altschul (1993) Applications and statistics for multiple high-scoring segments in molecular sequences. Proc. Natl. Acad. Sci. USA 90: 5873-5877.

Kawakami,A., Y. Sato and M. Yoshida (2008) Genetic engineering of rice capable of synthesizing fructans and enhancing chilling tolerance. J. Exp. Bot. 59: 793-802.

Kawasaki, T., K.Henmi, E. Ono, S. Hatakeyama, M.Iwano, H. Satoh and K. Shimamoto (1999) The small GTP-binding protein Rac is a regulator of cell death in plants. Proc. Natl. Acad. Sci. USA 96: 10922-10926.

Keller, F. and D.M. Pharr (1996) Metabolism of carbohydrates in sinks and sources: galactosyl-sucrose oligosaccharides. In: Zamski, E. and A.A. Schaffer (eds.) Photoassimilate Distribution in Plants and Crops: Source-Sink Relationships, Marcel Dekker, New York, pp. 157-183.

Kerr, P.S., R.W.Pearlstein, B.J.Schweiger, M.F. Becker-Manley and J.W.Pierce (1997) Nucleotide sequences of galactinol synthase from zucchini and soybean, U.S. Patent 5648210.

Liu, J.J.J., D.C.Krenz, A.F. Galvez and B.O. de Lumen (1998) Galactinol synthase (GS): increased enzyme activity and levels of mRNA due to cold and desiccation. Plant Sci. 134: 11-20.

Liu, K., L.Wang, Y.Xu, N.Chen, Q.Ma, F.Li and K.Chong (2007) Overexpression of OsCOIN, a putative cold inducible zinc finger protein, increased tolerance to chilling, salt and drought, and enhanced proline level in rice. Planta 226: 1007-1016.

Lou, Q., L.Chen, Z.Sun, Y.Xing, J.Li, X.Xu, H.Mei and L.Luo (2007) A major QTL associated with cold tolerance at seedling stage in rice (Oryza sativa L.). Euphytica 158: 87-94.

Mochida,K., K. Kawaura， E. Shimosaka， N. Kawakami， T. Shin-I, Y.Kohara, Y.Yamazaki and Y.Ogihara (2006) Tissue expression map of a large number of expressed sequence tags and its application to in silico screening of stress response genes in common wheat. Mol. Genet. Genomics 276: 304-312.

Morsy, M.R., L. Jouve, J.-F. Hausman, L. Hoffmann and J.McD. Stewart (2007) Alteration of oxidative and carbohydrate metabolism under abiotic stress in two rice (Oryza sativa L.) genotypes contrasting in chilling tolerance. J. Plant Physiol. 164: 157-167.

Nishiyama, I. (1985) Physiology of cool-weather damages in rice plant. Hokkaido University Press, Sapporo, p. 328.

Nishizawa,A., Y.Yabuta and S. Shigeoka (2008) Galactinol and raffinose constitute a novel function to protect plants from oxidative damage. Plant Physiol. 147: 1251-1263.

Obendorf, R.L., S. Odorcic, T. Ueda, M.P. Coseo and E. Vassallo (2004) Soybean galactinol synthase forms fagopyritol B1 but not galactopinitols: substrate feeding of isolated embryos and heterologous expression. Seed Sci. Res. 14: 321-333. 
Ogiwara, H. and K. Terashima (2001) A varietal difference in coleoptile growth is correlated with seedling establishment of direct seeded rice in submerged field under low-temperature conditions. Plant Prod. Sci. 4: 166-172.

Ozawa, K., S. Murayama, A. Kobayashi-Uehara and H.Handa (2006) Overexpression of wheat mitochondrial uncoupling protein in rice plants confers tolerances to oxidative stresses promoted by exogenous hydrogen peroxide and low temperature. Mol. Breed. 18: 5156.

Panikulangara, T.J., E.-S. Gabriele, M.Wunderlich, H. Stransky and F. Schöffl (2004) Galactinol synthase1. A novel heat shock factor target gene responsible for heat-induced synthesis of raffinose family oligosaccharides in Arabidopsis. Plant Physiol. 136: 31483158 .

Peterbauer, T. and A. Richter (2001) Biochemistry and physiology of raffinose family oligosaccharides and galactosyl cyclitols in seeds. Seed Sci. Res. 11: 185-197.

Saito, M. and M. Yoshida (2011) Expression analysis of the gene family associated with raffinose accumulation in rice seedlings under cold stress. J. Plant Physiol. 168: 2268-2271.

Saitou, N. and M.Nei (1987) The neighbor-joining method: a new method for reconstructing phylogenetic trees. Mol. Biol. Evol. 4: 406-425.

Sambrook, J., E.F. Frisch and T. Maniatis (1989) Molecular cloning: A Laboratory Manual, 2nd edn. Cold Spring Harbor Laboratory Press, Cold Spring Harbor, New York.

Sato, Y., T. Murakami, H. Funatsuki, S. Matsuba, H. Saruyama and M. Tanida (2001) Heat shock-mediated APX gene expression and protection against chilling injury in rice seedlings. J. Exp. Bot. 52: $145-151$.

Shimosaka,E., T.Sasanuma and H.Handa (1999) A wheat coldregulated cDNA encoding an early light-inducible protein (ELIP): its structure, expression and chromosomal location. Plant Cell Physiol. 40: 319-325.

Sprenger, N. and F. Keller (2000) Allocation of raffinose family oligosaccharides to transport and storage pools in Ajuga reptans: the roles of two distinct galactinol synthases. Plant J. 21: 249-258.

Taji, T., C. Ohsumi， S. Iuchi， M. Seki， M.Kasuga, M.Kobayashi, K. Yamaguchi-Shinozaki and K. Shinozaki (2002) Important roles of drought- and cold-inducible genes for galactinol synthase in stress tolerance in Arabidopsis thaliana. Plant J. 29: 417-426.

Takahashi, R., N. Joshee and Y. Kitagawa (1994) Induction of chilling resistance by water stress, and cDNA sequence analysis and expression of water stress-regulated genes in rice. Plant Mol. Biol. 26: $339-352$.

Thao, N.P., L. Chen, A. Nakashima, S. Hara, K. Umemura, A. Takahashi, K.Shirasu, T. Kawasaki and K. Shimamoto (2007) RAR1 and HSP90 form a complex with Rac/Rop GTPase and function in innate-immune responses in rice. Plant Cell 19: 4035-4045.

Toki, S. (1997) Rapid and efficient Agrobacterium-mediated transformation in rice. Plant Mol. Biol. Rep. 15: 16-21.

Ueda, T., M.P.Coseo, T.J.Harrell and R.L.Obendorf (2005) A multifunctional galactinol synthase catalyzes the synthesis of fagopyritol A1 and fagopyritol B1 in buckwheat seed. Plant Sci. 168: 681690.

Volk, G.M., E.E.Haritatos and R. Turgeon (2003) Galactinol synthase gene expression in melon. J. Am. Soc. Hortic. Sci. 128: 8-15.

Wang,Z., Y.Zhu, L.Wang, X.Liu, Y.Liu, J.Phillips and X.Deng (2009) A WRKY transcription factor participates in dehydration tolerance in Boea hygrometrica by binding to the W-box elements of the galactinol synthase (BhGolS1) promoter. Planta 230: 11551166.

Xie, L., Z. Tan, Y.Zhou, R. Xu, L. Feng, Y.Xing and X. Qi (2014) Identification and fine mapping of quantitative trait loci for seed vigor in germination and seedling establishment in rice. J. Integr. Plant Biol. 56: 749-759.

Yoshida, M., J.Abe, M.Moriyama and T.Kuwabara (1998) Carbohydrate levels among winter wheat cultivars varying in freezing tolerance and snow mold resistance during autumn and winter. Physiol. Plant. 103: 8-16.

Yoshida, M. and K. Tamura (2011) Research on fructan in wheat and temperate forage grasses in Japan. Jpn. Agric. Res. Q. 45: 9-14.

Zhang, Z.H., L. Su, W.Li, W. Chen and Y.-G.Zhu (2005) A major QTL conferring cold tolerance at the early seedling stage using recombinant inbred lines of rice (Oryza sativa L.). Plant Sci. 168: 527-534.

Zhao, T.-Y., R. Thacker, J.W. Corum III, J.C.Snyder, R.B. Meeley, R.L. Obendorf and B.Downie (2004) Expression of the maize GALACTINOL SYNTHASE gene family: (I) Expression of two different genes during seed development and germination. Physiol. Plant. 121: 634-646.

Zhuo, C., T.Wang, S.Lu, Y.Zhao, X.Li and Z.Guo (2013) A cold responsive galactinol synthase gene from Medicago falcata (MfGolS1) is induced by myo-inositol and confers multiple tolerances to abiotic stresses. Physiol. Plant. 149: 67-78. 